\title{
The role of oxidative stress in cancer associated with viral infection
}

\author{
Ewa Kliszczewska', Malgorzata Strycharz-Dudziak², Malgorzata Polz-Dacewicz' \\ ${ }^{1}$ Department of Virology, Medical University, Lublin, Poland \\ ${ }^{2}$ Chair and Department of Conservative Dentistry with Endodontics, Medical University, Lublin, Poland
}

Kliszczewska E, Strycharz-Dudziak M, Polz-Dacewicz M. The role of oxidative stress in cancer associated with viral infection. J Pre-Clin Clin Res. 2018; 12(2): 41-44. doi: 10.26444/jpccr/92218

\begin{abstract}
Introduction. The role of oxidative stress in the pathogenesis of neoplastic diseases, including its connection with viral infections, is the subject of many publications. The results of numerous researches have revealed that virus-induced phagocyte activation is associated with oxidative stress, not only because reactive oxygen species (ROS) are released, but also because activated phagocytes can release cytokines, such as tumour necrosis factor (TNF-alpha) or interleukin-1 (IL-1). Objective. The purpose of this review is to analyze the role of ROS in the pathogenesis of head and neck cancer and correlation between ROS and viruses, especially Epstein-Barr virus (EBV) and Human papillomavirus (HPV), in malignant tumour development in this area.

State of knowledge. The effects of an increased amount of ROS or reactive nitrate species (RNS) with a simultaneous reduction of antioxidants are noticed in various cancers, including head and neck cancer. Increased oxidative stress is also associated with disorders in the antioxidant defence system. HPV and EBV, which are important risk factors for head and neck cancer, can act via ROS-based mechanisms. Long-term expression of the Epstein-Barr nuclear antigen 1 (EBNA1) causes increased ROS and nicotinamide adenine dinucleotide phosphate (NADPH) oxidase level. During expression of HPV16infected cells, RNS increase E6 and E7 levels, thereby increasing the level of DNA damage in the cell. ROS are also involved in many benign oral disorders.

Conclusion. Reactive oxygen species are involved in various pathological processes in the environment of chronic oxidative stress, including carcinogenicity. ROS contribute to the development of head and neck cancer through many risk factors, such as connection with viruses.
\end{abstract}

\section{Key words}

head and neck cancer, oxidative stress, EBV, HPV

\section{INTRODUCTION}

The aerobic life of organisms, including humans, is associated with a kind of paradox - the organisms cannot exist without oxygen, while on the other hand, oxygen can be dangerous for them [1]. As revealed by Paul Bert [2] in the nineteenth century, oxygen in high concentrations can damage to the brain, lungs and other organs. In the human body, oxygen is used in metabolic processes to produce the necessary energy. Energy centres of eukaryotic cells are mitochondria producing numerous enzymes involved in respiratory chain pathways. One of the stages of cellular respiration is the four-stage electron transport chain in which oxygen is their acceptor. Sometimes, there is so-called "leakage of electrons from the respiratory chain" which result in incomplete full four-electron reduction of oxygen molecule. These disorders lead to formation of reactive oxygen species (ROS) [1].

\section{OBJECTIVE}

The aim of this review is to analyze the role of ROS in the pathogenesis of head and neck cancer and correlation between ROS and viruses, especially Epstein-Barr virus (EBV) and Human papillomavirus (HPV), in malignant

Adress for correspondence: Ewa Kliszczewska, Department of Virology, Medical University, Lublin, Poland

E-mail: ewakliszczewska@gmail.com

Received: 25 May 2018; Accepted: 13 June 2018 tumour development in this area. The review is based on literature research, using the PubMed database, taking into account papers published between 1990 - 2017 using searching terms "oxidative stress", "head and neck cancer" (585 records), and "oxidative stress" and "EBV” (58 records) and "oxidative stress" and "HPV" (81 records), "oxidative stress", "head and neck cancer", "EBV" (4 records), "oxidative stress", "head and neck cancer" and "HPV" (8 records). Finally, 31 items of synthetic original papers and reviews were selected which seemed to be the most useful.

Oxidative stress. Free radicals are molecules containing at least one unpaired electron on the outer electron shell. These molecules strive to pair electrons by taking them away or donating them to other molecules, which results in their high reactivity. Formation of free radicals is strongly influenced by physical factors, such as ionizing radiation, ultraviolet radiation, ultrasounds or elevated temperature.

The respiratory chain is the source of about $90 \%$ of free radicals [3]. The constant presence of ROS in low concentrations in the body helps the organism to properly run many life processes [1]. ROS participate in cellular functions such as intracellular signal transduction, proliferation, cell apoptosis, control over internal homeostasis of calcium $\mathrm{Ca}^{2+}$, regulation of gene expression, and participate in protein phosphorylation processes; they may also induce transcription factors (nuclear factor-kappa B - NF-kB), which causes disorders in the normal functioning of cells $[3,4]$. These molecules are also involved in the elimination of microorganisms [1]. ROS and 
reactive nitrate species (RNS) have a dual nature - on the one hand, they are necessary for normal cellular functions, but on the other hand, in excess they can damage cells and can lead to cancer development [5]. Free radicals may also damage all of the biomolecules found in the body at the molecular level and cell organelles. A significant role in the defene against free radicals is attributed to the activity of such enzymes as superoxide dismutase (SOD), catalase (CAT), glutathione peroxidase (GPx), glutathione reductase (GR), glutathione S-transferase (GST) and a group of secretory phospholipases A2 (sPLA2). GST plays an important role in detoxification processes, protecting cells from DNA damage, but it can also affect cancer cells protecting them against the toxicity of anticancer drugs $[1,3]$. Disorders in the balance between the formation of reactive oxygen species and the body's antioxidant capacity are called oxidative stress [3].

Markers of oxidative stress. Some of the most active ROS include the hydroxyl radical $(\mathrm{OH} \bullet)$ and superoxide radical ().

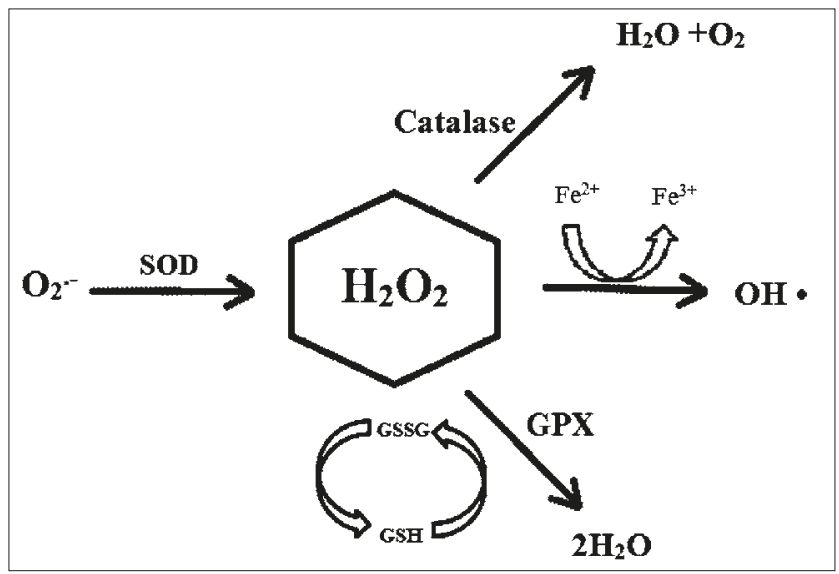

Figure 1. Balance between ROS and antioxidants [6]

It is estimated that in the respiratory chain about $2 \%$ of molecular oxygen is transformed into, which has the ability to oxidize transition metal ions. As a result, there may be a decreased activity of enzymes in which metals are found in active centres. This radical also has the ability to oxidize cysteine, which can lead to change of the protein conformation and cause loss of their biological properties. In the presence of SOD, the superoxide radical can be reduced to hydrogen peroxide $\left(\mathrm{H}_{2} \mathrm{O}_{2}\right) \cdot \mathrm{H}_{2} \mathrm{O}_{2}$ is not a free radical; however, as a result of the Fenton reaction in the presence of reduced forms of copper or iron cations, may be transformed into the most reactive radical in biological systems $-\mathrm{OH} \bullet$ :

$$
\begin{gathered}
\mathrm{Cu}^{+} / \mathrm{Fe}^{2+}+\mathrm{H}_{2} \mathrm{O}_{2} \rightarrow \mathrm{Cu}^{2+} / \mathrm{Fe}^{3+}+\mathrm{OH}^{-}+\mathrm{OH}^{\cdot} \\
\mathrm{Cu}^{2+} / \mathrm{Fe}^{3+}+\mathrm{H}_{2} \mathrm{O}_{2} \rightarrow \mathrm{Cu}^{+} / \mathrm{Fe}^{2+}+\mathrm{O}_{2}^{-}+2 \mathrm{H}^{+} \\
\frac{2 \mathrm{H}_{2} \mathrm{O}_{2} \rightarrow \mathrm{OH}^{-}+\mathrm{OH}^{\bullet}+\mathrm{O}^{\bullet-}+2 \mathrm{H}^{+}}{}
\end{gathered}
$$

$\mathrm{H}_{2} \mathrm{O}_{2}$ also enters Haber-Weiss reaction, i.e. with radical, in which $\mathrm{OH} \bullet$ is also formed [1]. $\mathrm{OH} \bullet$ is very reactive and reacts very quickly with majority of biomolecules. Transformations with this radical are based on detaching hydrogen atoms from the molecules attacked. Due to these properties, $\mathrm{OH}$ • does not penetrate cells and react with the nearest molecules; however, these changes can lead to cell damage. Damages often occur in locations very distant from the place of $\mathrm{OH} \bullet$ attack, therefore, the originally created radical centre located in a specific fragment of the protein chain may be transferred within the molecule [3].

The role of ROS in cancer. Oxidative stress plays a role in many diseases, including inflammation states, diabetes, cardiovascular diseases or cancer. The results of studies in patients with overt pathology (diabetes as well as ischemic heart disease) allow the treatment of oxidative stress as an element connecting different directions of metabolic disorders in the mentioned diseases.

The role of free radicals and ROS in many cancers has been documented. Intermediate products of oxygen reduction attack DNA and other cellular components, such as lipids, proteins, leaving reactive compounds that in turn can react with DNA bases [5].

The effects of an increased amount of ROS or RNS with a simultaneous reduction of antioxidants are noticed in various cancers, including head and neck cancer. ROS can be produced from endogenous and exogenous substances. Endogenous sources involve mitochondria, cytochrome P450 metabolism, peroxisomes and activation of inflammatory cells. Exogenous sources include environmental factors, including non-genotoxic carcinogens, xenobiotics, ultrasounds and microwave radiation. Cancer development is a complex, multi-stage process involving initiation, promotion and progression. ROS take part in all these stages. In certain stages of carcinogenesis, the influence of oxidative stress is directly proportional to the type and reactivity of the radicals present. Initiation takes place when a normal cell supports DNA mutation, which was previously generated and then after the stage of DNA synthesis was fixed - this results in the production of cells with the mutation. Cancer development with the involvement of ROS is supported by the occurrence of oxidative DNA modifications in tumour tissues. The promotion is characterized by the production of identically mutated cells, through induction of cell proliferation and/or inhibition of apoptosis [7]. Oxidative stress at this stage plays an important role as it can stimulate the expansion of mutant cell clones by temporary modulation of genes involved in cell proliferation or cell death [8], and regulate the activity of certain transcription factors (e.g., $\mathrm{NFKB}$ ) that control cell growth and oncogenesis. This may lead to the activation of NFKB with subsequent induction of genes encoding apoptosis-inhibiting proteins $[5,7,9]$. Even low levels of oxidative stress can stimulate cell division in promotion stage and therefore promote tumour growth. It may be concluded that ROS production at this stage is the main mechanism for the promotion of ROS-related cancers. ROS is also involved in the progression - at this stage, the generation of large amounts of ROS may contribute to mutation, increase the amount of matrix metalloproteinases (MMPs) and damage local tissues. Increased levels of DNA modified due to oxidation can contribute to genetic instability and may be crucial in tumour metastases in developed cancer $[5,7]$.

Head and neck cancer is the sixth most frequently occurring malignant tumour in the world $[10,11]$. About 550,000 new cases are diagnosed, leading to about 380,000 deaths annually worldwide due to this cancer [12]. Malignant neoplasms arise from the epithelium of the nasal cavity, oral cavity, paranasal sinus, throat and larynx. There are several types of oral cancer, but about $90 \%$ are squamous 
cell carcinomas (SCC). The ethology of SCC is complex and involves many factors. [13, 14, 15].

Smoking and alcohol abuse are among the main risk factors for head and neck cancer. Chronic inflammation may cause cancer coupled with mechanisms based on ROS, which secreted by stimulated neutrophils, regulate the growth and progression of cancer by stimulating proto-oncogene expression and angiogenesis $[16,17]$. Increased oxidative stress is also associated with disorders in the antioxidant defense system [7]. Tobacco chewing as well as smoking causes the oxidant/antioxidant imbalance. The heat generated during smoking and $\mathrm{pH}$ change during tabaco chewing affects the formation and destabilization of free radicals [7]. An important role in the process of tumour formation in oral squamous cell carcinoma is also attributed to mutations in the suppressor gene p53, which occur in about $42-49 \%$ of patients with diagnosed cancer. The area of the floor of the mouth is exposed to squamous cell carcinoma development due to continuous salivation, this is so-called "salivary gutter" area, where carcinogens may interact [17].

Some researchers revealed that in cancer there was a decrease in the activity of enzymes protecting against ROS: GPx, SOD and others [17]. The polymorphism of the single mitochondrial nucleotide SOD enzyme, SOD2, occurs with an increased frequency in smoking patients with oral SCC (OSCC), thus showing a relationship between ROS and carcinogenesis. Differences in polymorphisms were also found in the manganese SOD gene (MnSOD) in patients with a history of alcohol abuse, who subsequently developed oesophageal cancer [16].

Correlation between ROS and viruses. Many researches analysed the role of viruses in the development of squamous cell carcinoma. The most frequently studied viruses included HPV, EBV and HerpesSimplex type 1 virus (HSV-1) $[15,18$, 19]. It is estimated that approximately $90 \%$ of all head and neck cancers are SCC. As revealed by many researches, the etiology of SCC is complex and multifactorial. Molecular and epidemiological studies provided evidence for the role of oncogenic viruses in the development of SCC $[14,15,20$, 21]. HPV has been recognized by the International Agency for Research on Cancer as an important carcinogen in humans, causing cancer in the head and neck region [22]. The first human virus with an attributable oncogenic potential was EBV. The relationship between EBV infection and the development of head and neck cancer has been reported by many researchers $[23,24,25]$.

Each of these viruses, which are important risk factors for head and neck cancer, can act via ROS-based mechanisms. Long-term expression of the Epstein-Barr nuclear antigen 1 (EBNA1) causes increased ROS and nicotinamide adenine dinucleotide phosphate (NADPH) oxidase level. Decreased EBNA1 protein expression in EBV-positive tumour cells results in a decreased ROS level $[16,26]$. The increase in oxidative stress causes both direct oxidative damage to DNA, and is involved in various signaling pathways that can lead to chromosomal aberrations and cell transformation [27]. The EBV protein family includes the latency-associated proteins -1 and 2 (LMP-1 and LMP-2) that play a crucial role in the development and progression of cancer. Domains of LMP-1 are required for the maximal activation of NF-kB, which is linked to the inhibition of apoptosis, a major way adopted by cancer cells for survival [28].
During HPV infection, the immune response of the host is limited because the virus infects the basal epithelial cells. They are protected against circulating immune cells during the initial stages of infection. Nevertheless, reactive oxygen species and reactive nitrogen species are important for the development of associated cancer. During expression of HPV16-infected cells, RNS increase the levels of E6 and E7, thereby increasing the level of DNA damage in the cell $[27,29]$. Oxidative stress causes oxidative damage to the host DNA, thus facilitating the integration of HPV DNA into subsequent cells [30]. Leukocytes migrate to the site of infection and release cytokines to initiate elimination of viral infections, such as tumour necrosis factor (TNF-alpha) and interleukin-1 (IL-1). Chronic inflammation increases the level of ROS and RNS, leading to the oxidation of proteins, lipids and DNA. High-risk HPV (HPV 16 and 18) E2 proteins move between the nucleus and the cytoplasm, and the occurrence of E2 on mitochondrial membranes increases the production of mitochondrial ROS, which is not stated in the low-risk HPV6 E2 protein [16, 31]. In contrast, expression of the short E6 protein isoform from high-risk HPV forms increased ROS level in both HPV-positive and HPV-negative cells, along with decreased expression of SOD2 and GPx [16].

\section{CONCLUSIONS}

In summary, although reactive oxygen species are needed in normal cellular processes, they are also involved in various pathological processes in the environment of chronic oxidative stress, including carcinogenesis. ROS contribute to the development of head and neck cancer not only through general mechanisms of DNA damage and protein modulation, but also through many risk factors, such as connection with viruses. ROS are also involved in many benign oral disorders. ROS and redox pathways are an interesting target for the development of appropriate therapies. The challenge will be to develop therapies that will not threaten normal cellular functions based on ROS or redox pathways, while reducing pathological processes caused by ROS and redox.

\section{REFERENCES}

1. Karpińska A, Gromadzka G. Oxidative stress and natural antioxidant mechanisms: the role in neurodegeneration. From molecular mechanisms to therapeutic strategies. Postepy Hig Med Dosw. 2013; 67: 43-53. In Polish

2. Bert P. La Pression barometrique, Libraire de l'Acad de Med. 1878; Paris.

3. Kulbacka J, Saczko J, Chwiłkowska A. Oxidative stress in cells damage processes. Pol. Merk. Lek. 2009; XXVII, 157, 44. In Polish

4. Ścibior-Bentkowska D, Czeczot H. Cancer cells and oxidative stress. Postepy Hig Med Dosw. (online). 2009; 63: 58-72. In Polish

5. Choudhari SK, Chaudhary M, Gadbail AR, Sharma A, Tekade S. Oxidative and antioxidative mechanisms in oral cancer and precancer: a review. Oral Oncol. 2014; 50(1): 10-8.

6. Sharma S, Shrivastav A, Shrivastav BR. Clinical evidences of oxidative stress as biomarker in various types of cancers: A review. IJPSR. 2014; 5(3): 657-665.

7. Katakwar P, Metgud R, Naik S, Mittal R. Oxidative stress marker in oral cancer: A review. J Cancer Res Ther. 2016; 12(2): 438-46.

8. Trueba GP, Sánchez GM, Giuliani A. Oxygen free radical and antioxidant defence mechanism in cancer. Front Biosci. 2004; 9: 2029-2044.

9. Fuchs-Tarlovsky V. Role of antioxidants in cancer therapy. Nutrition. 2013; 29(1):15-21.

10. Mascolo M, Siano M, Ilardi G, Russo D, Merolla F, De Rosa G, et al. Epigenetic Disregulation in Oral Cancer. Int J Mol Sci. 2012; 13(2): 2331-2353. 
11. Polz-Gruszka D, Macieląg P, Fołtyn S, Polz-Dacewicz M. Oral squamous cell carcinoma (OSCC) - molecular, viral and bacterial concepts. J PreClin Clin Res. 2014; 8(2): 61-66.

12. Fitzmaurice C, Allen C, Barber RM, Barregard L, Bhutta ZA, Brenner $\mathrm{H}$, et al. Global, Regional, and National Cancer Incidence, Mortality, Years of Life Lost, Years Lived With Disability, and Disability-Adjusted Life-years for 32 Cancer Groups, 1990 to 2015: A Systematic Analysis for the Global Burden of Disease Study. JAMA Oncol. 2017, 3(4), 524-548.

13. Blot WJ, McLaughlin JK, Winn DM, Austin DF, Greenberg RS, PrestonMartin S, et al. Smoking and drinking in relation to oral and pharyngeal cancer. Cancer Res. 1988; 48(11): 3282-3287.

14. Fakhry C, Westra WH, Li S, Cmelak A, Ridge JA, Pinto H, et al. Improved survival of patients with human papillomavirus-positive head and neck squamous cell carcinoma in a prospective clinical trial. J Natl Cancer Inst. 2008; 100(4): 261-269.

15. Hillbertz NS, Hirsch JM, Jalouli J, Jalouli MM, Sand L. Viral and molecular aspects of oral cancer. Anticancer Res. 2012; 32(10): 42014212 .

16. Kesarwala AH, Krishna MC, Mitchell JB. Oxidative stress in oral diseases. Oral Dis. 2016; 22(1): 9-18.

17. Konopka T, Gmyrek-Marciniak A, Kozłowski Z, Kaczmarek U, Wnukiewicz J. Antioxidant Capacity of Saliva in Patients with Periodontitis and Squamous Carcinoma of the Mouth Floor. Dent Med Probl. 2006; 43(3): 354-362. In Polish

18. Metgud R, Astekar M, Verma M, Sharma A. Role of viruses in oral squamous cell carcinoma. Oncol Rev. 2012; 6(2): e21.

19. Sand L, Jalouli J. Viruses and oral cancer. Is there a link? Microbes Infect. 2014; 16(5): 371-378.

20. Gillison ML, Koch WM, Capone RB, Spafford M, Westra WH, Wu L, et al. Evidence for a causal association between human papillomavirus and a subset of head and neck cancers. J Natl Cancer Inst. 2000; 92(9): 709-720.

21. Scully C. Oral cancer aetiopathogenesis; past, present and future aspects. Medicina Oral Patologia Oral Y Cirugia Bucal. 2011; 16(3): e306-e311.
22.IARC Monographs on the Evaluation of Carcinogenic Risks to Humans. International Agency for Research on Cancer: Lyon, France. 2007; pp. 222-230.

23. Acharya S, Ekalaksananan T, Vatanasapt P, Loyha K, Phusingha P, Promthet $\mathrm{S}$, et al. Association of Epstein-Barr virus infection with oral squamous cell carcinoma in a case-control study. J Oral Pathol Med. 2005; 44(4): 252-257.

24. Jalouli J, Ibrahim SO, Mehrotra R, Jalouli MM, Sapkota D, Larson PA, et al. Prevalence of viral (HPV, EBV, HSV) infections in oral submucous fibrosis and oral cancer from India. Acta Otolaryngol. 2010; 130(11): 1306-1311.

25. Jalouli J, Jalouli MM, Sapkota D, Ibrahim SO, Larson PA, Sand L. Human papilloma virus, herpes simplex and Epstein-Barr virus in oral squamous cell carcinoma from eight different countries. Anticancer Res. 2012; 32(2): 571-580.

26. Cao JY, Mansouri S, Frappier L. Changes in the nasopharyn-geal carcinoma nuclear proteome induced by the EBNA1 protein of EpsteinBarr virus reveal potential roles for EBNA1 in metas-tasis and oxidative stress responses. J Virol. 2012; 86(1): 382-394.

27. Williams VM, Filippova M, Filippov V, Payne KJ, Duerksen-Hughes P. Human papillomavirus type $16 \mathrm{E} 6{ }^{*}$ induces oxidative stress and DNA damage. J Virol. 2014; 88(12): 6751-6761.

28. Radhashree M. Viruses and Head and Neck Cancers. Head and Cancer Research. 2015; 1(1): 3.

29. Wei L, Gravitt PE, Song H, Maldonado AM, Ozbun MA. Nitric oxide induces early viral transcription coincident with increased DNA damage and mutation rates in human papillomavirus-infected cells. Cancer Res. 2009; 69(11): 4878-4884.

30. Williams VM, Filippova M, Soto U, Duerksen-Hughes PJ. HPV-DNA integration and carcinogenesis: putative roles for inflammation and oxidative stress. Future Virol. 2011; 6(1): 45-57.

31. Lai D, Tan CL, Gunaratne J, Quek LS, Nei W, Thierry F, et al. Localization of HPV-18 E2 at mitochondrial membranes induces ROS release and modulates host cell metabolism. PLoS ONE. 2013; 8(9): e75625. 